TRANSACTIONS OF THE

AMERICAN MATHEMATICAL SOCIETY

Volume 357, Number 2, Pages 695-708

S 0002-9947(04)03532-9

Article electronically published on March 12, 2004

\title{
FINITE TIME BLOW-UP FOR A DYADIC MODEL OF THE EULER EQUATIONS
}

\author{
NETS HAWK KATZ AND NATAŠA PAVLOVIĆ
}

\begin{abstract}
We introduce a dyadic model for the Euler equations and the Navier-Stokes equations with hyper-dissipation in three dimensions. For the dyadic Euler equations we prove finite time blow-up. In the context of the dyadic Navier-Stokes equations with hyper-dissipation we prove finite time blow-up in the case when the dissipation degree is sufficiently small.
\end{abstract}

\section{INTRODUCTION}

The purpose of the paper is to discuss a dyadic model for the unforced incompressible constant density Euler equations in three dimensions and to show conditions under which this model and certain dissipative modifications blow up.

The Euler equations are a system of equations for an unknown time-varying vector field $u$ on $\mathbf{R}^{3}$, the velocity field of a fluid and an unknown function $p$, its pressure. The equations are

$$
\frac{\partial u}{\partial t}+(u \cdot \nabla) u=-\nabla p
$$

together with the divergence free condition

$$
\nabla \cdot u=0
$$

We let $T$ be the $L^{2}$ orthogonal projection into divergence free vector fields, and applying it to the equation (1.1), we get

$$
\frac{\partial u}{\partial t}+T((u \cdot \nabla) u)=0
$$

Thus we think of the Euler equations as saying that the change in the velocity $u$ is given as a certain bilinear operator of $u$. Of course, the Euler equations conserve energy which is defined as $\|u\|_{L^{2}}^{2}$. More precisely, for every $t>0$ one has

$$
\|u(., t)\|_{L^{2}}=\left\|u_{0}\right\|_{L^{2}},
$$

with $u_{0}=u(x, 0)$. This follows from the identity

$$
\langle T((u \cdot \nabla) u), u\rangle=0,
$$

where $\langle$,$\rangle is the L^{2}$ pairing.

Some basic questions concerning Euler equations are still unsolved. For example, it is an open problem to find out if solutions of the Euler equations in 3 dimensions satisfying bounded energy condition (i.e., $\left\|u_{0}\right\|_{L^{2}}=C$ ) form singularities in finite

Received by the editors July 25, 2003.

2000 Mathematics Subject Classification. Primary 35Q30, 35Q35, 76B03.

(C)2004 American Mathematical Society 
time. However, the local in time existence of solutions for 3D Euler equations is known in the Sobolev space $H^{s}, s>\frac{5}{2}$; see, for example, [7] or [11].

The Euler equations describe the motion of an inviscid fluid. To describe a viscous fluid, one adds a viscosity term. The resulting equations

$$
\frac{\partial u}{\partial t}+(u \cdot \nabla) u=-\nabla p-(-\Delta) u,
$$

are called the Navier-Stokes equations.

Sometimes to interpolate these equations one can study a family of intermediate equations

$$
\frac{\partial u}{\partial t}+(u \cdot \nabla) u=-\nabla p-(-\Delta)^{\alpha} u
$$

where $\alpha>0$. These are called the Navier-Stokes equations with dissipation $\alpha$.

It is easy to see that classical solutions to the Navier-Stokes equations with dissipation $\alpha$ satisfy decay of energy, i.e., for every $t>0$ one has

$$
\|u(., t)\|_{L^{2}}^{2}=\left\|u_{0}\right\|_{L^{2}}^{2}-2 \int_{0}^{t} \nu\left\langle(-\Delta)^{\alpha} u, u\right\rangle .
$$

As in the case of the Euler equations the theory of the Navier-Stokes equations in three dimensions is far from being complete. The major open problem is the question of global existence of smooth solutions of the Navier-Stokes equations in 3 dimensions satisfying bounded energy condition, where by a smooth solution in $\mathbb{R}^{3}$ one means a solution to the Navier-Stokes equations such that $u(x, t) \in$ $C^{\infty}\left[\mathbb{R}^{3} \times \mathbb{R}^{+}\right]$. For the precise formulation of this open problem and a review of known results (in particular, local in time existence results, global existence results with small initial data and partial regularity results) see [4].

Many results which can be obtained about Euler and Navier-Stokes equations use principally the conservation (decay) of energy and the scaling properties of the nonlinear term $\langle T((u \cdot \nabla) u), u\rangle$, most notably partial regularity results for the Navier-Stokes equations of Caffarelli, Kohn and Nirenberg [2], and a criterion for loss of regularity for solutions of the Euler equations of Beale, Kato, and Majda [1. We discuss certain dyadic models for the equations which share these properties.

The dyadic model presented here is an infinite system of nonlinearly coupled ODEs. Each ODE illustrates time evolution of a wavelet coefficient which describes behavior of the velocity that is localized to a certain frequency range. Therefore, the dyadic model could be understood in a general context of Littlewood-Paley theory, which allows localization of a function into frequency ranges. Recently LittlewoodPaley theory was extensively used in studying the Navier-Stokes equations. One of the reasons why Littlewood-Paley techniques are useful in applications to fluid equations is because they allow paraproduct decomposition of the nonlinear term $\langle T((u \cdot \nabla) u), u\rangle$, which in turn helps isolate certain frequency interactions. For an overview of existence results for the Navier-Stokes equations that use such an approach see, for example, [3]. Also each ODE in our model reflects behavior of the velocity localized in space on a dyadic cube. Here we used a standard harmonic analysis approach of decomposing $\mathbb{R}^{3}$ into dyadic cubes. Using such a decomposition we study flows on the dyadic tree.

We first used dyadic models in our work on partial regularity for the NavierStokes equations with hyper-dissipation [8] where they were a helpful test case for our ideas. On the other hand, by following harmonic analysis techniques one can obtain existence results for dyadic models that correspond to the known existence 
results for the actual Euler and Navier-Stokes equations. This is possible thanks to the fact that our model equations share the energy conservation and scaling properties of the actual equations. An example of such a local existence result for the dyadic Euler equations is given in 5. Also we note that the dyadic models belong to a general class of shell models introduced by Gledzer [6] and Ohkitani and Yamada [10] and are used for investigating turbulence.

In this paper, we obtain blow-up for models corresponding to the Euler equations and to the Navier-Stokes equations with dissipation $\alpha$ with $\alpha<\frac{1}{4}$. Our results work because in our models energy travels always from low frequency to high frequency. However, in the Euler equations, one of the principal ways that energy travels through frequency space is by the way in which the flow deforms space, because it is incompressible. We believe that it is likely the study of geometric behavior could lead to a real understanding of the Euler and Navier-Stokes equations.

The paper is organized as follows. In section 2 we introduce dyadic models. In section 3 we give a blow-up result for the dyadic Euler equations. Then in section 4 we present a proof of finite time blow-up for the dyadic Navier-Stokes equations with small dissipation.

\section{DYADIC MODEL}

Here we shall introduce a dyadic model for the equations of fluid motion in three dimensions.

We define a dyadic cube in a standard way. A cube $Q$ in $\mathbb{R}^{3}$ is a dyadic cube if its side length is an integer power of $2,2^{l}$, and the corners of the cube are on the lattice $2^{l} \mathbb{Z}^{3}$.

We let $\mathcal{D}$ denote the set of dyadic cubes in $\mathbb{R}^{3}$. We let $\mathcal{D}_{j}$ denote the subset of dyadic cubes having side length $2^{-j}$. Abusing notation slightly we define the function

$$
j: \mathcal{D} \longrightarrow \mathbb{Z}
$$

by letting $j(Q)=j$ if $Q \in \mathcal{D}_{j}$. We define $\tilde{Q}$, the parent of $Q$, to be the unique dyadic cube in $\mathcal{D}_{j(Q)-1}$ which contains $Q$. For $m \geq 1$ we define $\mathcal{C}^{m}(Q)$, the $m$ th order grandchildren of $Q$ to be the set of those cubes in $\mathcal{D}_{j(Q)+m}$ which are contained in $Q$. We sometimes refer to the first order grandchildren of $Q$ as the children of $Q$.

In our dyadic model we consider a scalar-valued function $u(x, t)$. It is represented by a wavelet expansion

$$
u(x, t)=\sum_{Q} u_{Q}(t) w_{Q}(x),
$$

where $\left\{w_{Q}\right\}$ is an orthonormal family in $L^{2}\left(\mathbb{R}^{3}\right)$ of wavelets such that the wavelet $w_{Q}$ is localized at the spatial dyadic cube $Q \in \mathcal{D}_{j}$. The wavelet coefficient corresponding to the cube $Q$ is denoted by $u_{Q}(t)$. We will refer to the values $u_{Q}(t)$ as the coefficients of the function $u$.

We define the dyadic Laplacian $\Delta$ by

$$
\Delta\left(w_{Q}\right)=2^{2 j(Q)} w_{Q} .
$$

We define $\|u\|_{L^{2}}$ to denote the $L^{2}$ norm of $u$ and for any $\alpha>0$, we define

$$
\|u\|_{H^{2 \alpha}}=\|u\|_{L^{2}}+\left\|(\Delta)^{\alpha} u\right\|_{L^{2}} .
$$


We define $\langle u, v\rangle$ to denote the $L^{2}$ pairing of $u$ and $v$. Since our wavelets $w_{Q}$ are orthonormal in $L^{2}\left(\mathbb{R}^{3}\right)$, we notice that

$$
\langle u, v\rangle=\sum_{Q} u_{Q}(t) v_{Q}(t) .
$$

We would like to have an operator which will mimic the behavior of the nonlinear term $T(u \cdot \nabla u)$. We define a bilinear operator, the cascade operator, by defining two pieces from which it is built. The cascade down operator is defined by

$$
\left(C_{d}(u, v)\right)_{Q}=2^{\frac{5 j(Q)}{2}} u_{\tilde{Q}} v_{\tilde{Q}} .
$$

We define the cascade up operator by

$$
\left(C_{u}(u, v)\right)_{Q}=2^{\frac{5(j(Q)+1)}{2}} u_{Q} \sum_{Q^{\prime} \in C^{1}(Q)} v_{Q^{\prime}} .
$$

We define the cascade operator

$$
C(u, v)=C_{u}(u, v)-C_{d}(u, v) .
$$

Obviously,

$$
\left\langle C_{u}(u, u), u\right\rangle=\left\langle C_{d}(u, u), u\right\rangle
$$

which implies

$$
\langle C(u, u), u\rangle=0 .
$$

The choice of the exponent $\frac{5}{2}$ comes from the scaling properties of $u \cdot \nabla u$. A heuristic motivation is as follows. If $w_{Q}$ were an $L^{2}$ normalized (vector-valued) wavelet supported on $Q \in \mathcal{D}_{j}$, it would have

$$
\left\|w_{Q}\right\|_{L^{\infty}} \sim 2^{\frac{3 j}{2}}
$$

Moreover, it would satisfy

$$
\left\|\nabla w_{Q}\right\|_{L^{\infty}} \sim 2^{\frac{5 j}{2}}
$$

since differentiating adds a factor of $2^{j}$. Thus one would have

$$
\left|w_{Q} \cdot \nabla w_{Q}\right| \lesssim 2^{4 j} \lesssim 2^{\frac{5 j}{2}}|| w_{Q}||_{L^{\infty}} .
$$

Having defined operator $C(u, v)$ we can speak about the dyadic version of the Euler as well as the Navier-Stokes equations. More precisely, we shall say that a time varying function $u$ satisfies the dyadic Euler equations provided that

$$
\frac{d u}{d t}+C(u, u)=0 .
$$

Also we shall say that $u$ satisfies the dyadic Navier-Stokes equations with hyperdissipation if

$$
\frac{d u}{d t}+C(u, u)+(\Delta)^{\alpha} u=0
$$

We will restrict our attention to functions $u$ all of whose coefficients $u_{Q}$ are initially nonnegative. This class of functions is preserved by both flows (2.3) and (2.4). Let us verify that for the flow (2.3). Fix a dyadic cube $Q$. We rewrite the equation (2.3) in terms of wavelet coefficients as follows:

$$
\frac{d u_{Q}(t)}{d t}+2^{\frac{5(j(Q)+1)}{2}} u_{Q} \sum_{Q^{\prime} \in C^{1}(Q)} u_{Q^{\prime}}(t)=2^{\frac{5 j(Q)}{2}} u_{\tilde{Q}^{2}}^{2}(t) .
$$


Now we remark that the equation (2.5) is a first order linear ordinary differential equation in $u_{Q}(t)$ and its solution is

$$
u_{Q}(t)=\frac{1}{\mu(t)}\left(u_{Q}(0)+\int_{0}^{t} 2^{\frac{5 j(Q)}{2}} u_{\tilde{Q}}^{2}(\tau) \mu(\tau) d \tau\right),
$$

where

$$
\mu(t)=e^{\int_{0}^{t} 2^{\frac{5(j(Q)+1)}{2}} \sum_{Q^{\prime} \in C^{1}(Q)} u_{Q^{\prime}}(\tau) d \tau .}
$$

Since $\mu(t)>0$ for all $t$, (2.6) implies that $u_{Q}(t) \geq 0$ for all $t>0$, provided that $u_{Q}(0)$ is nonnegative.

\section{The Dyadic Euler equations}

3.1. Energy flow. One of the most important features of the flow (2.3) is that it conserves energy. To be more precise,

$$
\frac{d}{d t}(\langle u, u\rangle)=0 .
$$

This can be obtained by pairing (2.3) with $u$. Energy can be thought of as divided up amongst the nodes $Q$. To be more precise, if we write

$$
E=\langle u, u\rangle,
$$

then

$$
E=\sum_{Q \in \mathcal{D}} E_{Q}
$$

where

$$
E_{Q}=u_{Q}^{2}
$$

The flow (2.3) gives rise to an extremely local description of energy flow along the tree $\mathcal{D}$.

To be precise

$$
\frac{d}{d t} E_{Q}=E_{Q, i n}-E_{Q, o u t}
$$

where

and

$$
E_{Q, i n}=2^{\frac{5 j(Q)}{2}}\left(2 E_{\tilde{Q}} \sqrt{E_{Q}}\right),
$$

$$
E_{Q, \text { out }}=\sum_{Q^{\prime} \in \mathcal{C}^{1}(Q)} E_{Q^{\prime}, \text { in }} .
$$

Thus energy is flowing always from larger squares to smaller ones and indeed it flows along the edges of the tree $\mathcal{D}$.

We define a Carleson box by

$$
\mathcal{C}(Q)=\bigcup_{k=1}^{\infty} \mathcal{C}^{k}(Q)
$$

and the energy of a Carleson box by

$$
E_{\mathcal{C}(Q)}=\sum_{Q^{\prime} \in \mathcal{C}(Q)} E_{Q^{\prime}} .
$$

Also we shall introduce an extended Carleson box by

$$
\mathcal{C}_{0}(Q) \equiv Q \cup \mathcal{C}(Q)
$$


Then we define the energy of $\mathcal{C}_{0}(Q)$ by

$$
E_{\mathcal{C}_{0}(Q)}=\sum_{Q^{\prime} \in \mathcal{C}_{0}(Q)} E_{Q^{\prime}}
$$

By using (2.1) we sum (3.2) over all cubes $Q \in \mathcal{C}(Q)$ and $Q \in \mathcal{C}_{0}(Q)$ respectively, to obtain the following proposition.

Proposition 3.1.1. Let $u$ be a time-varying "function" with positive coefficients evolving according to the flow (2.3). Then for any $Q$, the functions in time given by $E_{\mathcal{C}(Q)}$ and $E_{\mathcal{C}_{0}(Q)}$ are monotone increasing.

3.2. The heart. We begin with an easy lemma about Carleson boxes.

Lemma 3.2.1. For any $\epsilon>0$, there is $\delta(\epsilon)>0$, so that if we know that

$$
E_{\mathcal{C}(Q)}>(1-\delta) 2^{-(3+\epsilon) j(Q)},
$$

then there exists $Q^{\prime} \in \mathcal{C}^{1}(Q)$, so that

$$
E_{\mathcal{C}_{0}\left(Q^{\prime}\right)} \geq 2^{-(3+\epsilon) j\left(Q^{\prime}\right)} .
$$

Proof. Let us suppose the conclusion of the lemma is false, i.e.,

$$
E_{\mathcal{C}_{0}\left(Q^{\prime}\right)}<2^{-(3+\epsilon) j\left(Q^{\prime}\right)},
$$

for all $Q^{\prime} \in \mathcal{C}^{1}(Q)$.

On the other hand, we know

$$
E_{\mathcal{C}(Q)}=\sum_{Q^{\prime} \in \mathcal{C}^{1}(Q)} E_{\mathcal{C}_{0}\left(Q^{\prime}\right)} .
$$

Now because we are in dimension 3 , there are exactly $2^{3}$ elements $Q^{\prime} \in \mathcal{C}^{1}(Q)$ with $j\left(Q^{\prime}\right)=j(Q)+1$. Thus (3.4) combined with (3.3) implies

$$
E_{\mathcal{C}(Q)} \leq 2^{3} \cdot 2^{-(3+\epsilon)(j(Q)+1)},
$$

and therefore,

$$
E_{\mathcal{C}(Q)} \leq 2^{-\epsilon} \cdot 2^{-(3+\epsilon) j(Q)}
$$

which is a contradiction provided we have chosen $\delta$ sufficiently small such that $2^{-\epsilon}<1-\delta$.

Now we prove the main lemma.

Lemma 3.2.2. Fix $j_{0}$ sufficiently large. Then there is a sufficiently small $0<\epsilon<1$ so that if at time $t_{0}$ we have

$$
E_{\mathcal{C}_{0}(Q)} \geq 2^{-(3+\epsilon) j(Q)},
$$

with $j(Q)>j_{0}$, then there is some $t$ with $t<t_{0}+2^{-\epsilon j(Q)}$ and a cube $Q^{\prime} \in \mathcal{C}^{1}(Q)$ so that at time $t$ we have $E_{\mathcal{C}_{0}\left(Q^{\prime}\right)} \geq 2^{-(3+\epsilon) j\left(Q^{\prime}\right)}$.

Proof. We assume that the conclusion of the lemma is false, i.e.,

$$
E_{\mathcal{C}_{0}\left(Q^{\prime}\right)}<2^{-(3+\epsilon) j\left(Q^{\prime}\right)},
$$

for all $Q^{\prime} \in \mathcal{C}^{1}(Q)$ and for all $t \in\left[t_{0}, t_{0}+2^{-\epsilon j(Q)}\right]$.

In light of Lemma 3.2.1 and Proposition 3.1.1 it must be the case that for all $t \in\left[t_{0}, t_{0}+2^{-\epsilon j(Q)}\right]$ we have $E_{Q} \geq \delta 2^{-(3+\epsilon) j(Q)}$, since otherwise because of the 
hypothesis (3.5) we would have $E_{\mathcal{C}(Q)}>(1-\delta) 2^{-(3+\epsilon) j(Q)}$, which would by Lemma 3.2.1 lead to a contradiction.

Moreover, since energy flows only in the direction of smaller cubes and is conserved, it must be that for any child $Q^{\prime} \in \mathcal{C}^{1}(Q)$, it is the case that

$$
E_{\mathcal{C}(Q)} \geq \int_{t_{0}}^{t_{0}+2^{-\epsilon j(Q)}} E_{Q^{\prime}, i n}
$$

at time $t_{0}+2^{-\epsilon j(Q)}$. Thus since

$$
E_{Q^{\prime}, i n}=2^{\frac{5(j(Q)+1)}{2}} 2 E_{Q} u_{Q^{\prime}}
$$

we must have

$$
\int_{t_{0}}^{t_{0}+2^{-\epsilon j(Q)}} u_{Q^{\prime}} \lesssim \frac{1-\delta}{\delta} 2^{\frac{-5 j(Q)}{2}} .
$$

However, we know that

$$
\frac{d u_{Q^{\prime}}}{d t}=2^{\frac{5(j(Q)+1)}{2}} E_{Q}-2^{\frac{5(j(Q)+2)}{2}} u_{Q^{\prime}}\left(\sum_{Q^{\prime \prime} \in \mathcal{C}^{1}\left(Q^{\prime}\right)} u_{Q^{\prime \prime}}\right) .
$$

Thus integrating, applying the fact that for all $t \in\left[t_{0}, t_{0}+2^{-\epsilon j(Q)}\right]$, we have $E_{Q}>$ $\delta 2^{-(3+\epsilon) j(Q)}$, and $u_{Q^{\prime \prime}} \lesssim 2^{-\frac{3 j(Q)}{2}}$ (we can afford to give back the $\epsilon$ ), and using (3.6), we see that the first term of (3.7) dominates (for $\epsilon$ sufficiently large and $j(Q)$ sufficiently small) and

$$
\int_{t_{0}}^{t_{0}+2^{-\epsilon j(Q)}} \frac{d u_{Q^{\prime}}}{d t} \gtrsim \delta 2^{-\left(\frac{1}{2}+2 \epsilon\right) j(Q)},
$$

which is a contradiction of our upper bound on $u_{Q^{\prime}}$ by the fundamental theorem of calculus.

Corollary 3.2.3. Let $u$ be a solution to (2.3) which has initially all positive coefficients, and at time 0 has $E_{Q}>2^{-(3+\epsilon) j(Q)}$, for some $Q$ with $j(Q)>j_{0}$ with $j_{0}$ as in the previous lemma. Then the $H^{\frac{3}{2}+\epsilon}$ norm of $u$ becomes unbounded in finite time.

Proof. We apply the lemma. We find a cube $Q_{1}$ properly contained in $Q$ and a time $t_{1}<2^{-\epsilon j(Q)}$ so that at $t_{1}$ we have $E_{\mathcal{C}_{0}\left(Q_{1}\right)}>2^{-(3+\epsilon) j\left(Q_{1}\right)}$.

We iterate this procedure finding a cube $Q_{k}$ properly contained in $Q_{k-1}$ and a time $t_{k}$ so that $t_{k-1} \leq t_{k}<t_{k-1}+2^{-\epsilon j\left(Q_{k-1}\right)}$ and at time $t_{k}$ we have $E_{\mathcal{C}_{0}\left(Q_{k}\right)}>$ $2^{-(3+\epsilon) j\left(Q_{k}\right)}$.

Estimating just using the coefficients of $\mathcal{C}_{0}\left(Q_{k}\right)$, we see that at time $t_{k}$, we have that

$$
\begin{aligned}
\|u\|_{H^{\frac{3}{2}+\epsilon}}^{2} & \geq \sum_{j \geq j\left(Q_{k}\right)} 2^{(3+2 \epsilon) j} \sum_{Q \in \mathcal{D}_{j}} u_{Q}^{2}\left(t_{k}\right) \\
& \geq 2^{(3+2 \epsilon) j\left(Q_{k}\right)} \sum_{j \geq j\left(Q_{k}\right)} \sum_{Q \in \mathcal{D}_{j}} u_{Q}^{2}\left(t_{k}\right) \\
& \geq 2^{(3+2 \epsilon) j\left(Q_{k}\right)} E_{\mathcal{C}_{0}\left(Q_{k}\right)}\left(t_{k}\right) \\
& \geq 2^{\epsilon j\left(Q_{k}\right)} .
\end{aligned}
$$


Since $j\left(Q_{k}\right)$ is an increasing sequence of integers, this is going to $\infty$. However, since

$$
t_{k}=\left(t_{k}-t_{k-1}\right)+\left(t_{k-1}-t_{k-2}\right)+\cdots+t_{1} \leq 2^{-\epsilon j(Q)}+\sum_{l=1}^{k-1} 2^{-\epsilon j\left(Q_{l}\right)}
$$

and the $j\left(Q_{l}\right)$ 's are an increasing sequence of integers, we see that the sequence $\left\{t_{k}\right\}$ converges to a finite limit.

We point out here that under certain assumptions on the initial $E_{Q}(0)$ Corollary 3.2 .3 guarantees finite time blow-up of $\|u\|_{H^{\frac{3}{2}+\epsilon}}$. This was obtained using an extended Carleson box $\mathcal{C}_{0}(Q)$. In the next section we shall prove for the Navier-Stokes equations with small dissipation, finite time blow-up of slightly weaker $\|u\|_{H^{2+\epsilon}}$ norm. The reason for this is the fact that for the Navier-Stokes equations with hyper-dissipation the energy itself is not conserved. Instead part of it dissipates at each level of our dyadic tree $\mathcal{D}$.

However, from Fedor Nazarov [9] we learned from his proof that the solution to the dyadic Navier-Stokes equations with enough dissipation stays bounded in a certain $C^{k}$ space provided that it started in the same $C^{k}$ space. The main tool in his proof is the following observation. Let us truncate the system of ODEs which describe the dyadic Navier-Stokes equations with hyper-dissipation. If provided with enough dissipation, the system will scatter all energy. Therefore, energy cannot become concentrated over the first few levels. For example, this is true for the dyadic Navier-Stokes itself. A natural question is: what does "enough dissipation" mean? In the following section we prove that if the dissipation exponent $\alpha$ is less than $\frac{1}{4}$, one obtains finite time blow-up.

\section{The Dyadic NAVIER-Stokes Equations With HyPER-Dissipation}

4.1. Energy flow. We consider the dyadic Navier-Stokes equations with hyperdissipation (2.4). Since

$$
\langle C(u, u), u\rangle=0
$$

we have

$$
\frac{1}{2} \frac{d}{d t}(\langle u, u\rangle)+\left\langle(\Delta)^{\alpha} u, u\right\rangle=0
$$

and therefore, we have energy decay

$$
\langle u, u\rangle+2 \int_{0}^{t}\left\langle(\Delta)^{\alpha} u, u\right\rangle=\left\langle u_{0}, u_{0}\right\rangle
$$

where $u_{0}=u(x, 0)$.

Let us imagine that each node $Q$ along our tree $\mathcal{D}$ has a wastebasket which is at time $t$ filled with $2 \int_{t_{0}}^{t} 2^{2 \alpha j(Q)} u_{Q}^{2}$.

We define the energy of a cube $Q$ at time $t$ as in the case of the dyadic Euler equation

$$
E_{Q}(t)=u_{Q}^{2}(t)
$$

For $t$ greater than or equal to some fixed time $t_{0}$ we introduce the energy of a wastebasket of a cube $Q$ at time $t$ as

$$
W_{Q, t_{0}}(t)=2 \int_{t_{0}}^{t} 2^{2 \alpha j(Q)} E_{Q}
$$


Then (4.1) is saying that the sum of energy at nodes plus energy at wastebaskets is controlled.

Also we have a local description of energy flow along the tree $\mathcal{D}$ :

$$
\frac{d}{d t} E_{Q}=E_{Q, \text { in }}-E_{Q, o u t}-2^{2 \alpha j(Q)} 2 E_{Q}
$$

where

$$
E_{Q, i n}=2^{\frac{5 j(Q)}{2}} 2 E_{\tilde{Q}} \sqrt{E_{Q}}
$$

and

$$
E_{Q, \text { out }}=\sum_{Q^{\prime} \in \mathcal{C}^{1}(Q)} E_{Q^{\prime}, \text { in }} .
$$

Thus energy is flowing always from larger squares to smaller ones and, except for portions which go to wastebaskets, energy flows along the edges of the tree $\mathcal{D}$.

We define the energy of a Carleson box by

$$
E_{\mathcal{C}(Q)}=\sum_{Q^{\prime} \in \mathcal{C}(Q)} E_{Q^{\prime}},
$$

and the waste of a Carleson box by

$$
W_{\mathcal{C}(Q), t_{0}}=\sum_{Q^{\prime} \in \mathcal{C}(Q)} W_{Q^{\prime}, t_{0}} .
$$

We immediately get the following proposition.

Proposition 4.1.1. Let $u$ be a time-varying "function" with positive coefficients evolving according to the flow (2.4). Then for any $Q$, the function in time given by $E_{\mathcal{C}(Q)}+W_{\mathcal{C}(Q), t_{0}}$ is monotone increasing.

4.2. Energy concentration. As in the Euler case we begin with a lemma about Carleson boxes.

Lemma 4.2.1. For any $\epsilon>0$, there is $\delta(\epsilon)>0$, so that if we know that

$$
E_{\mathcal{C}(Q)}+W_{\mathcal{C}(Q), t_{0}}>(1-\delta) 2^{-(4+\epsilon) j(Q)},
$$

then there exists $Q^{\prime} \in \mathcal{C}(Q)$, so that

$$
E_{Q^{\prime}}+W_{Q^{\prime}, t_{0}} \geq 2^{-(4+\epsilon) j\left(Q^{\prime}\right)} .
$$

Proof. Let us assume the lemma is false, i.e.,

$$
E_{Q^{\prime}}+W_{Q^{\prime}, t_{0}}<2^{-(4+\epsilon) j\left(Q^{\prime}\right)},
$$

for all $Q^{\prime} \in \mathcal{C}(Q)$.

On the other hand, we have

$$
\begin{aligned}
E_{\mathcal{C}(Q)}+W_{\mathcal{C}(Q), t_{0}} & =\sum_{Q^{\prime} \in \mathcal{C}(Q)}\left[E_{Q^{\prime}}+W_{Q^{\prime}, t_{0}}\right] \\
& =\sum_{k=1}^{\infty} \sum_{Q^{\prime} \in \mathcal{C}^{k}(Q)}\left[E_{Q^{\prime}}+W_{Q^{\prime}, t_{0}}\right]
\end{aligned}
$$

Now because we are in dimension 3 , there are exactly $2^{3 k}$ elements $Q^{\prime} \in \mathcal{C}^{k}(Q)$ with $j\left(Q^{\prime}\right)=j(Q)+k$. Thus by using (4.3) we can bound (4.4) from above by

$$
\sum_{k=1}^{\infty} 2^{3 k} \cdot 2^{-(4+\epsilon)(j(Q)+k)}
$$


which is in turn the same as

$$
2^{-(4+\epsilon) j(Q)} \sum_{k=1}^{\infty} 2^{-(\epsilon+1) k} .
$$

Since

$$
\sum_{k=1}^{\infty} 2^{-(\epsilon+1) k}<1
$$

we can choose $0<\delta<1$ such that

$$
\sum_{k=1}^{\infty} 2^{-(\epsilon+1) k}<1-\delta .
$$

Therefore, (4.5) is bounded above by $(1-\delta) 2^{-(4+\epsilon) j(Q)}$, and we obtain

$$
E_{\mathcal{C}(Q)}+W_{\mathcal{C}(Q), t_{0}}<(1-\delta) 2^{-(4+\epsilon) j(Q)}
$$

which contradicts the assumption of our lemma.

Now we prove the main lemma.

Lemma 4.2.2. Fix $j_{0}$ sufficiently large. Then there exists an $\epsilon, 0<\epsilon<1-4 \alpha$, so that if at time $t_{0}$, we have

$$
E_{Q}+W_{Q, t_{0}} \geq 2^{-(4+\epsilon) j(Q)}
$$

with $j(Q)>j_{0}$, then there is some $t$ with $t<t_{0}+T$, where

$$
2^{\frac{(\epsilon-1) j(Q)}{2}}<T<2^{-2 \alpha j},
$$

and a cube $Q^{\prime} \in \mathcal{C}(Q)$ so that at time $t$ we have

$$
E_{Q^{\prime}}+W_{Q^{\prime}, t_{0}} \geq 2^{-(4+\epsilon) j\left(Q^{\prime}\right)} .
$$

Proof. We assume that the conclusion of the lemma is false, i.e.,

$$
E_{Q^{\prime}}+W_{Q^{\prime}, t_{0}}<2^{-(4+\epsilon) j\left(Q^{\prime}\right)},
$$

for all $Q^{\prime} \in \mathcal{C}(Q)$, and for all $t \in\left[t_{0}, t_{0}+T\right]$.

In light of Lemma 4.2.1 and Proposition 4.1.1 it must be the case that for all $t \in\left[t_{0}, t_{0}+T\right]$, we have

$$
E_{Q}(t)+W_{Q, t_{0}}(t) \geq \delta 2^{-(4+\epsilon) j(Q)}
$$

since otherwise because of the hypothesis 4.6 we would have

$$
E_{\mathcal{C}(Q)}+W_{\mathcal{C}(Q)}>(1-\delta) 2^{-(4+\epsilon) j(Q)} \text {, at some } t \in\left[t_{0}, t_{0}+T\right],
$$

which would lead to a contradiction by Lemma 4.2.1.

Since $W_{Q, t_{0}}(t)$ is a monotone increasing function of $t$, 4.8 implies that

$$
E_{Q}(t)+W_{Q, t_{0}}\left(t_{0}+T\right) \geq \delta 2^{-(4+\epsilon) j(Q)}, \text { for all } t \in\left[t_{0}, t_{0}+T\right],
$$

and therefore, we have either

$$
E_{Q}(t) \geq \frac{1}{2} \delta 2^{-(4+\epsilon) j(Q)}, \text { for all } t \in\left[t_{0}, t_{0}+T\right],
$$

or

$$
W_{Q, t_{0}}\left(t_{0}+T\right) \geq \frac{1}{2} \delta 2^{-(4+\epsilon) j(Q)} .
$$

We shall analyze those cases separately. 
First let us assume (4.10). Let $Q^{\prime}$ be an element of $\mathcal{C}^{1}(Q)$. Then $u_{Q^{\prime}}$ satisfies

$$
\frac{d u_{Q^{\prime}}}{d t}=2^{\frac{5(j(Q)+1)}{2}} u_{Q}^{2}-2^{\frac{5(j(Q)+2)}{2}} u_{Q^{\prime}}\left(\sum_{Q^{\prime \prime} \in \mathcal{C}^{1}\left(Q^{\prime}\right)} u_{Q^{\prime \prime}}\right)-2^{2 \alpha(j(Q)+1)} u_{Q^{\prime}} .
$$

We shall integrate (4.11) on the time interval $\left[t_{0}, t_{0}+T\right]$.

In order to simplify our notation we introduce the following integrals:

$$
\begin{aligned}
I_{1} & :=\int_{t_{0}}^{t_{0}+T} 2^{\frac{5(j(Q)+1)}{2}} u_{Q}^{2}, \\
I_{2} & :=\int_{t_{0}}^{t_{0}+T} 2^{\frac{5(j(Q)+2)}{2}} u_{Q^{\prime}}\left(\sum_{Q^{\prime \prime} \in \mathcal{C}^{1}\left(Q^{\prime}\right)} u_{Q^{\prime \prime}}\right), \\
I_{3} & :=\int_{t_{0}}^{t_{0}+T} 2^{2 \alpha(j(Q)+1)} u_{Q^{\prime}} .
\end{aligned}
$$

By using (4.10) we estimate $I_{1}$ and obtain

$$
I_{1}:=\int_{t_{0}}^{t_{0}+T} 2^{\frac{5(j(Q)+1)}{2}} u_{Q}^{2} \gtrsim \delta 2^{-\left(\frac{3}{2}+2 \alpha+\epsilon\right) j}
$$

We bound $I_{2}$ as follows:

$$
\begin{aligned}
I_{2} & :=\int_{t_{0}}^{t_{0}+T} 2^{\frac{5(j(Q)+2)}{2}} u_{Q^{\prime}}\left(\sum_{Q^{\prime \prime} \in \mathcal{C}^{1}\left(Q^{\prime}\right)} u_{Q^{\prime \prime}}\right) \\
& \leq \int_{t_{0}}^{t_{0}+T} 2^{\frac{5(j(Q)+2)}{2}} u_{Q^{\prime}}\left|\sum_{Q^{\prime \prime} \in \mathcal{C}^{1}\left(Q^{\prime}\right)} u_{Q^{\prime \prime}}\right| \\
& \lesssim 2^{\frac{5 j}{2}} \cdot T \cdot 2^{-(4+\epsilon) j} \cdot 2^{3},
\end{aligned}
$$

where the last inequality follows from (4.7). Thus

$$
I_{2} \lesssim T \cdot 2^{-\left(\frac{3}{2}+\epsilon\right) j} .
$$

Similarly, we use (4.7) in order to get

$$
I_{3}:=\int_{t_{0}}^{t_{0}+T} 2^{2 \alpha(j(Q)+1)} u_{Q^{\prime}} \lesssim T \cdot 2^{\left(2 \alpha-2-\frac{\epsilon}{2}\right) j} .
$$

One easily checks from (4.12), (4.13) and 4.14) that if $0<\epsilon<1-4 \alpha$ and $T<2^{-2 \alpha j}$, then

$$
I_{1} \geq I_{2},
$$

as well as

$$
I_{1} \geq I_{3}
$$

Therefore, after integrating (4.11) on the time interval $\left[t_{0}, t_{0}+T\right]$ we conclude that

$$
\int_{t_{0}}^{t_{0}+T} \frac{d u_{Q^{\prime}}}{d t} \gtrsim \delta 2^{-\left(\frac{3}{2}+2 \alpha+\epsilon\right) j},
$$

which is a contradiction by the fundamental theorem of calculus, since $\epsilon<1-4 \alpha$.

Now we are left to verify the contradiction in the case that one has (4.9). Let us assume (4.9). Moreover, since energy flows only in the direction of smaller cubes 
and is conserved up to waste baskets, it must be that for any child $Q^{\prime} \in \mathcal{C}^{1}(Q)$, it is the case that

$$
E_{\mathcal{C}(Q)}+W_{\mathcal{C}(Q), t_{0}} \geq \int_{t_{0}}^{t_{0}+T} E_{Q^{\prime}, i n}
$$

at time $t_{0}+T$. Thus since

$$
E_{Q^{\prime}, i n}=2^{\frac{5(j(Q)+1)}{2}} 2 E_{Q} u_{Q^{\prime}}
$$

we must have

$$
\int_{t_{0}}^{t_{0}+T} u_{Q^{\prime}} \lesssim \frac{1-\delta}{\delta} 2^{\frac{-5 j(Q)}{2}} .
$$

We integrate (4.11) on the time interval $\left[t_{0}, t_{0}+T\right]$. By using (4.9) we bound $I_{1}$ as follows:

$$
I_{1}:=\int_{t_{0}}^{t_{0}+T} 2^{\frac{5(j(Q)+1)}{2}} u_{Q}^{2} d t \gtrsim T \cdot \delta \cdot 2^{-\left(\frac{3}{2}+\epsilon\right) j} .
$$

We bound $I_{2}$ as

$$
\begin{aligned}
I_{2} & :=\int_{t_{0}}^{t_{0}+T} 2^{\frac{5(j(Q)+2)}{2}} u_{Q^{\prime}}\left(\sum_{Q^{\prime \prime} \in \mathcal{C}^{1}\left(Q^{\prime}\right)} u_{Q^{\prime \prime}}\right) \\
& \leq \int_{t_{0}}^{t_{0}+T} 2^{\frac{5(j(Q)+2)}{2}} u_{Q^{\prime}}\left|\sum_{Q^{\prime \prime} \in \mathcal{C}^{1}\left(Q^{\prime}\right)} u_{Q^{\prime \prime}}\right| \\
& \lesssim \frac{1-\delta}{\delta} 2^{-\left(2+\frac{\epsilon}{2}\right) j},
\end{aligned}
$$

where the last inequality follows from (4.15) and (4.7). Thus

$$
I_{2} \lesssim \frac{1-\delta}{\delta} 2^{-\left(2+\frac{\epsilon}{2}\right) j} .
$$

By using (4.15) we obtain the following bound on $I_{3}$

$$
I_{3}:=\int_{t_{0}}^{t_{0}+T} 2^{2 \alpha(j(Q)+1)} u_{Q^{\prime}} \lesssim 2^{\left(2 \alpha-\frac{5}{2}\right) j} .
$$

Again one easily checks from (4.16), (4.17) and (4.18) that $I_{1}$ dominates provided that $0<\epsilon<1-4 \alpha$ and $T>2^{\frac{(\epsilon-1) j}{2}}$.

Therefore, after integrating (4.11) on the time interval $\left[t_{0}, t_{0}+T\right]$ we conclude that

$$
\int_{t_{0}}^{t_{0}+T} \frac{d u_{Q^{\prime}}}{d t} \gtrsim T \cdot \delta \cdot 2^{-\left(\frac{3}{2}+\epsilon\right) j}
$$

which is a contradiction by the fundamental theorem of calculus, since $T>2 \frac{(\epsilon-1) j(Q)}{2}$.

Lemma 4.2.3. Fix $j_{0}$ sufficiently large. Then there exists an $\epsilon, 0<\epsilon<1-4 \alpha$, such that if at time $t_{0}$ we have

$$
E_{Q} \gtrsim 2^{-(4+\epsilon) j(Q)}
$$

with $j(Q)>j_{0}$, then there is some time $t$ with $t<t_{0}+T$, where

$$
2^{\frac{(\epsilon-1) j(Q)}{2}}<T<2^{-2 \alpha j(Q)},
$$


and a cube $Q^{\prime} \in \mathcal{C}(Q)$ so that at time $t$ we have

$$
E_{Q^{\prime}} \gtrsim 2^{-(4+\epsilon) j\left(Q^{\prime}\right)} \text {. }
$$

Proof. We shall prove the lemma by contradiction. Assume the lemma is false, i.e.,

$$
E_{Q^{\prime}}<2^{-(4+\epsilon) j\left(Q^{\prime}\right)} \text {, for all } Q^{\prime} \in \mathcal{C}(Q) \text {, and for all } t \in\left[t_{0}, t_{0}+T\right] .
$$

However, from the hypothesis (4.19) and Lemma 4.2.2 we can find time $t_{1}<$ $t_{0}+T$, where

$$
2^{\frac{(\epsilon-1) j(Q)}{2}}<T<2^{-2 \alpha j(Q)}
$$

and a cube $Q_{1} \in \mathcal{C}(Q)$ so that

$$
E_{Q_{1}}\left(t_{1}\right)+W_{Q_{1}, t_{0}}\left(t_{1}\right) \geq 2^{-(4+\epsilon) j\left(Q_{1}\right)} .
$$

On the other hand, by using monotonicity of the function $W_{Q_{1}, t_{0}}(t)$ and (4.20) we calculate

$$
\begin{aligned}
E_{Q_{1}}\left(t_{1}\right)+W_{Q_{1}, t_{0}}\left(t_{1}\right) & <E_{Q_{1}}\left(t_{1}\right)+W_{Q_{1}, t_{0}}\left(t_{0}+T\right) \\
& =E_{Q_{1}}\left(t_{1}\right)+2 \int_{t_{0}}^{t_{0}+T} 2^{2 \alpha j\left(Q_{1}\right)} E_{Q_{1}} \\
& <2^{-(4+\epsilon) j\left(Q_{1}\right)}+2 \cdot 2^{2 \alpha j\left(Q_{1}\right)} \cdot 2^{-(4+\epsilon) j\left(Q_{1}\right)} \cdot T \\
& \lesssim 2^{-(4+\epsilon) j\left(Q_{1}\right)},
\end{aligned}
$$

where the last inequality follows from (4.21).

Thus

$$
E_{Q_{1}}\left(t_{1}\right)+W_{Q_{1}, t_{0}}\left(t_{1}\right) \lesssim 2^{-(4+\epsilon) j\left(Q_{1}\right)},
$$

which contradicts 4.22), and the lemma is proved.

Corollary 4.2.4. Let $u$ be a solution to (2.4) which has all positive coefficients and at time 0 has some $Q$ with $E_{Q}>2^{-(4+\epsilon) j(Q)}$, for $j(Q)>j_{0}$ with $j_{0}$ as in the previous lemma. Then the $H^{2+\epsilon}$ norm of $u$ becomes unbounded in finite time.

Proof. We apply Lemma 4.2.3. We find a cube $Q_{1}$ properly contained in $Q$ and a time $t_{1}<T<2^{-2 \alpha j(Q)}$ so that at $t_{1}$ we have $E_{Q_{1}} \gtrsim 2^{-(4+\epsilon) j\left(Q_{1}\right)}$.

We iterate this procedure finding a cube $Q_{k}$ properly contained in $Q_{k-1}$ and a time $t_{k}$ so that $t_{k-1} \leq t_{k}<t_{k-1}+T<t_{k-1}+2^{-2 \alpha j\left(Q_{k-1}\right)}$, and at time $t_{k}$ we have $E_{Q_{k}} \gtrsim 2^{-(4+\epsilon) j\left(Q_{k}\right)}$.

Estimating just using the coefficient at $Q_{k}$, we see that at time $t_{k}$ we have that

$$
\|u\|_{H^{2+\epsilon}} \geq 2^{(2+\epsilon) j\left(Q_{k}\right)} E_{Q_{k}}^{1 / 2} \geq 2^{\frac{\epsilon j\left(Q_{k}\right)}{2}} .
$$

Since $j\left(Q_{k}\right)$ is an increasing sequence of integers, this is going to $\infty$. However, since

$$
t_{k}=\left(t_{k}-t_{k-1}\right)+\left(t_{k-1}-t_{k-2}\right)+\cdots+t_{1} \leq 2^{-2 \alpha j(Q)}+\sum_{l=1}^{k-1} 2^{-2 \alpha j\left(Q_{l}\right)},
$$

and the $j\left(Q_{l}\right)$ 's are an increasing sequence of integers, we see that the sequence $\left\{t_{k}\right\}$ converges to a finite limit. 


\section{ACKNOWLEDGEMENTS}

The first author was partially supported by NSF grant number DMS 0100601. The second author acknowledges support from the Clay Mathematics Institute and NSF grant number DMS 0304594. We would both like to thank the Mathematics Department of Princeton University for its hospitality in the Spring of 2001. We would like to thank Susan Friedlander and Fedor Nazarov for helpful discussions.

\section{REFERENCES}

[1] J.T. Beale, T. Kato, and A. Majda: Remarks on the breakdown of smooth solutions for the $3 D$ Euler equations, Comm. Math. Phys. 94 , No. 1 (1984), 61-66. MR 85j:35154

[2] L. Caffarelli, R. Kohn, and L. Nirenberg: Partial regularity of suitable weak solutions of the Navier-Stokes equations, Comm. Pure Appl. Math. 35, No. 6 (1982), 771-831. MR 84m:35097

[3] M. Cannone: Harmonic analysis tools for solving the incompressible Navier-Stokes equations, To appear in Handbook of Mathematical Fluid Dynamics 3 (2004).

[4] C. Fefferman: Existence and smoothness of the Navier-Stokes equation, http://www. claymath.org, (2000).

[5] S. Friedlander, and N. Pavlović: Blow up in a three-dimensional vector model for the Euler equations, Preprint, (2003).

[6] E. B. Gledzer: System of hydrodynamic type admitting two quadratic integrals of motion, Sov. Phys. Dokl. 18, No. 4 (1973), 216-217.

[7] Kato, T.: Quasi-linear equations of evolution with applications to partial differential equations, Lecture Notes in Mathematics 448, Berlin, Heidelberg, New York, Springer, ( 1975), 25-70. MR 53:11252

[8] N.H. Katz and N. Pavlović: A cheap Caffarelli-Kohn-Nirenberg inequality for the NavierStokes equation with hyper-dissipation, GAFA 12 (2002), 355-379. MR 2003e:35243

[9] F. Nazarov: Personal communication, (2001).

[10] K. Ohkitani and M. Yamada: Temporal intermittency in the energy cascade process and local Lyapunov analysis in fully developed model turbulence, Prog. Theor. Phys. 81, No. 2 (1989), 329-341. MR 90j:76065

[11] R. Temam: Local existence of $C^{\infty}$ solutions of the Euler equations of incompressible perfect fluids, Lecture Notes in Mathematics 565, Berlin, Heidelberg, New York,Sp ringer, (1976), 184-195. MR 57:6902

Department of Mathematics, Washington University, St. Louis, Missouri 63130

E-mail address: nets@math.wustl.edu

Department of Mathematics, Princeton University, Princeton, New Jersey 08544

E-mail address: natasa@math.princeton.edu 